\title{
Sobre la situación de las plantas faenadoras de carne en
}

\section{Chile.}

\author{
María Pía Meledandri \\ Municiplaidad de Traiguén/Pragrama PDTI INDAP, Unidad Operativa Comunal, \\ Territorio 1. Email: pia mele@hotmail.com
}

Recibido: 23 de mayo de 2017; Aceptado: 06 de junio de 2017.

En 1563 se estableció el primer matadero en Chile, "Matadero San Miguel". A partir del año 1868 la labor de fiscalización, reglamentación y funcionamiento administrativo de mataderos atañó al municipio correspondiente según jurisdicción hasta la década del 60 y gracias a la constante presión ejercida por los organismos sanitarios oficiales se delegó al médico veterinario administrador, las tareas de inspección médico veterinaria (Luengo, J. 1998). En el año 1968 se publicó en el Diario Oficial el Código Sanitario, aludiendo la responsabilidad al Servicio Nacional de Salud (SNS) todas las áreas relacionadas con salud pública (MINSAL 1968).

Más tarde en el año 1994 entró en vigencia la Ley 19.162 o Ley de Carnes que "establece el sistema obligatorio de clasificación de ganado, tipificación y nomenclatura de sus carnes y regula el funcionamiento de mataderos, frigoríficos y establecimientos de la industria de la carne" (SAG, s.f). Estableciendo las labores de fiscalización y control de dichas actividades al Servicio Agrícola y Ganadero (SAG). Y en el año 2002 se aprobó la norma general técnica no. 62 sobre inspección médico-veterinaria de reses de abasto y de sus carnes y criterios para la calificación de aptitud para el consumo humano estableciendo los procedimientos de inspección y criterios de aptitud para las carnes en Chile (Viveagro 2010).

El 23 de agosto del año 2010 el Servicio de Salud delegó las funciones de inspección médico veterinaria de los animales de abasto y sus carnes en mataderos al SAG, entidad hoy responsable de declarar la aptitud para consumo humano de las carnes y subproductos (Carmona, 2010).

La inspección de carnes es una disciplina sujeta a cambios para satisfacer las necesidades de los consumidores y cumplir con la normativa vigente (Moreno, 2006). Para Ávila (2007) las buenas prácticas de manufactura (BPM) son el fundamento sanitario por el que las empresas dedicadas a manufacturar alimentos deben regirse 
para obtener un producto de calidad e inocuo. Las BPM deben ser documentadas en forma de manuales que permitan guiar y tomar decisiones, así como también deben incluir los procedimientos operacionales estandarizados (SOP) y los procedimientos operacionales estandarizados de sanitización (SSOP), teniendo como principal objetivo la prevención de posibles alteraciones del producto antes, durante y después de su procesamiento y distribución (SAG 1999).

En la actualidad, el control de los alimentos en Chile ha adquirido una gran relevancia debido principalmente a la globalización que ha alcanzado el comercio nacional e internacional de alimentos y la importancia dada por los propios consumidores a la inocuidad alimentaria (Universidad de Chile s.f.).

\section{REFERENCIAS}

Ávila, ML, 2007, 'Diseño de la Documentación del Sistema de Buenas Prácticas de Manufactura para la Empresa Productos Le Chandelier', Licenciada en Tecnología de Alimentos, Escuela de tecnología de Alimentos, Facultad de Ciencias Agroalimentarias, Universidad de Costa Rica.

Carmona, K 2010, 'Servicio de Salud traspasa la inspección de mataderos al SAG', Chile: alimentos inocuos Chile: food safety [Blog], 23 agosto de 2010 consultado: 5 de abril de 2012, http://chilealimentosinocuos.blogspot.com/2010/08/servicio-de-salud-traspasala.html.

Luengo, J 1998, 'Los mataderos en Chile antes de la aplicación de la ley 19.162' [En línea], Revista Tecno Vet, Vol. 4, No 1, consultado: 15 mayo de 2012, http://www. revistas.uchile.cl/index.php/RT/article/view/5210/5092.

Ministerio de Salud (MINSAL) 1968, Código Sanitario, D.F.L. No. 725/67, Ministerio de Salud, República de Chile.

Moreno, B 2006, Higiene e inspección de carnes - I, $2^{\circ}$ Ed., editorial Díaz de Santos, España.

Servicio Agrícola y ganadero (SAG) 1999, Manual Genérico Sistemas de Aseguramiento de Calidad, Proyecto no. 322, Ministerio de Agricultura, República de Chile, pp. 6-7.

Viveagro 2010, SAG asume funciones de inspección veterinaria en 42 nuevos mataderos y establecimientos de Faena [Página web], consultado: 5 junio de 2013, http://www.viveagro.cl/index.php/sag-asume-funciones-de-inspeccionveterinaria-en-42 -nuevos-mataderos-y-establecimientos-de-faena/. 
Sustainability, Agri, Food and Environmental Research, 5(2), 2017: 43-44 ISSN: 0719-3726 